\title{
New life after near death: Surviving critical COVID-19 infection
}

\author{
B Hodkinson, ${ }^{1} \mathrm{PhD}, \mathrm{MB}$ BCh; P Gina, ${ }^{2} \mathrm{MB}$ BCh, HIV Dip (SA), MMed; M Schneider, ${ }^{3} \mathrm{PhD}, \mathrm{MA}, \mathrm{BSc}$ \\ ${ }^{1}$ Rheumatology Division, Department of Medicine, Groote Schuur Hospital, University of Cape Town, South Africa \\ ${ }^{2}$ Division of Pulmonology, Department of Medicine, Groote Schuur Hospital, University of Cape Town, South Africa \\ ${ }^{3}$ Alan J Flisher Centre for Public Mental Health, University of Cape Town, South Africa
}

Corresponding author: B Hodkinson (drbridget@gmail.com)

\begin{abstract}
Background. Few studies have explored the illness perceptions, experiences or attitudes towards the future of survivors of critical coronavirus disease 2019 (COVID-19). Through in-depth qualitative interviews, we aimed to enrich our understanding of participants' perspectives, with the hope of offering more holistic and appropriate care to future patients.

Methods. Participants who had survived critical COVID-19 illness (defined as a laboratory or clinical diagnosis of COVID-19, with hypoxia requiring high-flow nasal oxygen (HFNO) or mechanical ventilation) were invited to participate. After informed consent procedures, clinic-demographic details were documented and individual interviews conducted using a topic guide, and were audio-recorded, translated, transcribed and coded into NVivo software where themes were extracted.

Results. Of 21 participants ( 13 female, 8 male), the mean age was 51.8 years (range $34-68$ ), and mean duration of COVID symptoms was 21.7 days (range 17 - 37). Eighteen participants had been on HFNO, and 5 required mechanical ventilation. The major themes were: distressing experience; faith-based beliefs sustaining them; gratitude to healthcare workers (HCWs); better understanding of COVID and how dangerous it is; optimism for the future; and a resolve to implement lifestyle changes.

Conclusion. Qualitative interviews revealed our participants' experience of severe COVID-19 as a difficult and terrifying ordeal, mitigated by faith-based beliefs, and the presence and care of HCWs. These experiences were reported by the participants as life changing, and all were inspired to focus on future self-care, and invest in fulfilling relationships. These insights call for future interventions to improve patientcentred care, including follow-up debriefing sessions, and support for lifestyle changes.

Keywords. patient experiences; perceptions; COVID-19.
\end{abstract}

Afr J Thoracic Crit Care Med 2021;27(4):151-155. https://doi.org/10.7196/AJTCCM.2021.v27i4.184

From May to September 2020, Groote Schuur Hospital (GSH), a tertiary care hospital in South Africa, faced the first surge of coronavirus disease 2019 (COVID-19) admissions, with 50 - 60 sick adults admitted daily. Therapy for this disease is supportive, and hypoxic patients are prescribed corticosteroids, anticoagulation and oxygen offered at the lowest possible fraction of inspired oxygen necessary to keep peripheral oxygen saturation above 90\%. Low-flow oxygen can be delivered by nasal prongs, venturi face mask or nonrebreather mask at up to $20 \mathrm{~L} / \mathrm{min}$. Patients with higher oxygen needs ('critical COVID') may be offered high-flow nasal oxygen (HFNO) or mechanical ventilation in high care or an intensive care unit (ICU). Critical COVID-19 has a very high mortality: of 293 patients offered HFNO at GSH, 156 (53\%) failed HFNO, and 129 (82.7\%) of these patients died. ${ }^{[1]}$ Similar very high mortality rates have been reported elsewhere. ${ }^{[2,3]}$

On admission to GSH, patients were triaged according to their oxygen requirements, prognosis and eligibility for ICU admission. ${ }^{[4]}$ During this first wave, 46 HFNO and 36 ICU beds were available. As patients improved and their oxygen requirements decreased, they were transferred from high care and ICU beds to general COVID wards and, once stable, were discharged to field hospitals or home.Since the outbreak of this pandemic, thousands of papers and reviews have been published focusing on the epidemiology and spread of the virus, screening, testing and vaccination procedures, immune response and mechanisms of inflammation, and outcomes and management strategies. A few studies have explored the illness perceptions, experiences during critical illness or the impact of COVID-19 on perspectives in survivors of critical COVID-19 disease. These studies describe both positive (e.g. life-affirming effect of surviving) and negative aspects (e.g. fear and pain) of the experience. ${ }^{[5-7]}$ A recent systematic review of patient and family experiences of ICU admission highlighted the limited number of studies reflecting patients' experiences. ${ }^{[8]}$ There is good evidence to support the value of qualitative studies in epidemic and pandemic research to capture psychological and social aspects of the illness. ${ }^{[9,10]}$ By interviewing patients surviving critical COVID disease, and recording the stories of their illness, we aimed to enrich our understanding of patients' experiences, their understanding of the illness and their future lives, with the hope of offering patientcentred care and support to future patients. Ethical clearance was obtained from the University of Cape Town Human Research Ethics Committee (ref. no. HREC 419/2020).

\section{Participants and methods}

We conducted a qualitative study in severe or critical COVID-19 illness, defined as a laboratory or clinical diagnosis of COVID-19 with 
hypoxia requiring HFNO or mechanical ventilation. Between July and September 2020, patients surviving this high-care episode who had been transferred to general COVID wards at GSH were invited to participate. Confused or agitated participants were excluded.

In-depth semi-structured individual interviews were conducted by one of the authors, a clinician working in the COVID wards. Interviews took place in the ward when participants had minimal or no oxygen requirements and felt comfortable to talk, on their day of discharge from hospital. All participants signed informed consent to the interview, including audio-recording. Demographic information, details of hospital admission and critical illness course, and comorbidities were documented. A topic guide was used to explore experiences of the disease, particularly highlighting perceptions of COVID-19 before becoming ill, experiences while on HFNO or ICU including near-death phenomena, coping mechanisms, feelings about disclosing the coronavirus illness to family and community, and ideas of what the future might look like. This interview guide was tested in a pilot study of four participants and found to be satisfactory. Interviews took between 10 and 20 minutes in English or Afrikaans, and were audio-recorded, translated and transcribed into English. Interviews were stopped when saturation was reached after 21 interviews were completed. After each interview, audio data were transcribed and coded into NVivo software (QSR International, Australia) for sorting and extraction of themes. Participants are quoted in their own words, noting that many are not first-language English speakers.

\section{Results}

Of 23 patients invited to participate, 21 agreed to the interview, with two declining because of discomfort or difficulty in talking. The mean (range) age of the 21 participants was 51.8 (34 - 68) years (Table 1). The mean (range) duration of COVID-19 symptoms was 21.7 (17 - 37) days. Five participants had no comorbidities, and three of these were under 40 years old. Eighteen participants

Table 1. Demographic and clinical data of the 21 study participants

\begin{tabular}{ll}
\hline Characteristics & $\boldsymbol{n}(\%)^{*}$ \\
\hline Age (years), mean (range) & $51.8(34-68)$ \\
$40-70$ & $16(76.2)$ \\
$18-40$ & $5(23.8)$ \\
Sex & \\
$\quad$ Female & $13(61.9)$ \\
Comorbidities & \\
Diabetes mellitus & $13(61.9)$ \\
Hypertension & $13(61.9)$ \\
HIV-positive & $4(19.0)$ \\
Ischaemic heart disease & $1(4.8)$ \\
Renal transplant & $1(4.8)$ \\
No comorbidities & $5(23.8)$ \\
Treatment & \\
HFNO & $18(85.7)$ \\
Ventilation & $5(23.8)$ \\
HIV = human immunodeficiency virus; HFNO = high-flow nasal oxygen. \\
*Unless otherwise specified.
\end{tabular}

survived HFNO, and all had a relatively short duration of HFNO (median 5.8 days of HFNO; range 1 - 23 days), and five required mechanical ventilation for a median of 17 days of ventilation (range 5 - 27 days).

The major themes identified were: distressing experience; faithbased beliefs sustaining them; gratitude to healthcare workers (HCWs); better understanding of COVID and how dangerous it is; optimism for the future; and resolve to implement lifestyle changes.

\section{Distressing nature of severe COVID illness}

All participants described their time in hospital as a harrowing and very difficult experience, and one that they would not like to experience again or wish on others. For most, the feeling of shortness of breath was the most distressing symptom, frequently described with graphic imagery.

'It felt like I was drowning.' [67 yr pensioner, HFNO $\times 4$ days]

'...it's something like ice blocks cracking my body.' [63 yr pensioner, $\mathrm{HFNO} \times 3$ days $]$

One participant explained that shortness of breath was his worst problem and the one that brought most anxiety, and described his feeling of helplessness.

'It was the shortness of breath that I couldn't handle. But I have to, at the end of the day I had to deal with it. I can't run away. I can't complain, I can't cry. I can't do anything.' [29 yr man, HFNO $\times 23$ days]

Eleven participants described their confusion and anxiety at the time of admission to hospital, and one explained:

'By the time I came here, I didn't even know my name. Everything, I didn't knowanything.' [34 yr woman, ventilated $\times 5$ days]

The majority (20/21) believed that they had been near to death. When asked directly, 10 participants described classic near-death and out-ofbody experiences including meeting a deceased relative or a spiritual figure, or seeing a brilliant light or tunnel.

'I believe I nearly died, because I felt it. It was like gripping onto something, but your hand slips every time.' [ $35 \mathrm{yr}$ woman, HFNO $\times 3$ days]

'I see my grandmother, the one that went away from me. It was like she was calling me, but I was like, no.' [29 yr man, HFNO $\times 23$ days]

Three participants described seeing participants around them die in the ward as disturbing and contributing to their fear and feeling of hopelessness.

'I was seeing people dying too, people die around me. Tomorrow morning you see them, then the brother is not there anymore ... It's scary.' $[29 \mathrm{yr}$ man, $\mathrm{HFNO} \times 23$ days $]$

Many participants described the loneliness of their COVID illness. Separation from family and disallowance of hospital visits was one of the worst burdens carried by participants. In addition, many felt that they had no control over their circumstances or the outcomes of their illness. 
'I felt alone. I was alone.' [58 yr woman, ventilated $\times 10$ days] 'I feel lonely and I felt helpless.' [ $57 \mathrm{yr}$ seamstress, HFNO $\times 12$ days]

Other participants described the medical team's constant presence as a comfort mitigating the isolation and loneliness.

'No, I don't feel alone. Every time the doctor came and check on me. All of them. Starting from ICU to the wards.' [49 yr domestic worker, ventilated 36 days]

Some participants described using their mobile phones as a way to stay in touch with family, bringing great consolation. One participant described daily phone calls from her eldest son, a prisoner, as a source of inspiration.

'My son is in jail. So that is the one who was worried about me. He talked to me daily. Mamma, are you alright now? I said I'm fine, my son.' [54 yr domestic worker, HFNO $\times 4$ days]

One participant enjoyed the support offered by a large social network. 'I had so much support from everyone around the country. All my Facebook friends, my WhatsApp, everybody. People were crying, texting.' [ 61 yr musician, $\mathrm{HFNO} \times 6$ days]

Others had no breath or no energy to interact. Two participants explained that they did not want to talk to their families because they felt the family would be unable to understand the situation and it was difficult to explain to them.

\section{Survival strategies}

The majority of participants described an active decision to fight the illness, and many felt this was a source of strength that helped them to survive.

'For me, I like to pull myself together and examine the situation. I'm not going to give up. It's my body. I'm not going to give up.' [54 yr teacher, $\mathrm{HFNO} \times 4$ days]

Six participants felt that faith-based beliefs had seen them through this ordeal. Many expressly stated that God gave them strength to survive. For some, their faith brought them great comfort and acceptance of any outcome.

I'm talking to my God. It's His choice. It's not my choice. It's not your choice. It's God's choice. If He feel you must die that day, then you have to go, but I talk to Him.' [64 yr pensioner, HFNO $\times 3$ days]

'I've got a great sacrifice in my heart. Patience, sacrifice. Whatever God put you through today, thank you' [64 yr pensioner, $\mathrm{HFNO} \times 3$ days]

Many participants reported that the medical team was the reason they survived.

'Prayer and the doctors. That is what I think. That God used you, the doctors, and everyone... He gave you the knowledge, wisdom and grace, to - now I'm talking about myself - to help me.' [57 yr seamstress, $\mathrm{HFNO} \times 9$ days]

Some felt that their sense of responsibility to their family, particularly children, kept them going.
'You have to survive. You think of your kids, your wife. That's what's holding you back. I just believed that I can't slip. They are too young.' [35 yr unemployed mother, HFNO $\times 3$ days]

'...Daddy, we know you are a fighter, go there please, and come back home. So, I promised. I never break my promise to my kids.' [45 yr electrician, $\mathrm{HFNO} \times 5$ days $]$

\section{Gratitude for care}

Participants were generally positive about the care they received and expressed gratitude to the medical teams. Many felt inspired and motivated by the encouragement and optimism of the nurses and doctors who were present day and night.

'The confidence the doctors put in you, it's 100\%. They always tell you, don't worry, you'll get better. You just have to believe it.' [42 yr shopfitter, HFNO $\times 3$ days]

'I will always remember it, doctor. The encouragement to never give up. Nobody gave up on me. I will always be grateful, and I will always pray for you.' [ $67 \mathrm{yr}$ pensioner, $\mathrm{HFNO} \times 4$ days]

'I would like to say to the nurses...you make us strong, thank you to the doctors, thank you to everybody. You were really looking after us.' [54 yr factory worker, HFNO $\times 6$ days]

'You might not remember me after this, but I will always remember you and I will never forget to pray for you, never.' [35 yr woman, $\mathrm{HFNO} \times 3$ days $]$

One participant described the Transcendental Meditation technique taught to her by one of the doctors as helpful.

'One of the doctors who sat there [when] I was panicking, because it felt like I couldn't breathe. She asked me where I'm coming from. I replied and said, I'm from Oudtshoorn... So, she told me close my eyes. I must forget about the oxygen and everything. I must just picture driving past the mountains and that helped me. She took my attention away from it all, from my current situation at the time. I'm thankful.' [67 yr pensioner, $\mathrm{HFNO} \times 4$ days]

Some reported that good luck, or fate, was on their side and led to their survival. One explained her survival as a miracle.

'Whatever comes my way, I'm happy. I went through a lot of things in my life.' [64 yr pensioner, HFNO $\times 3$ days]

Most participants felt that explanations from HCWs were good. A few expressed a need for more information from the medical team, and described the chaos in the admission ward or high care wards as overwhelming and frightening, with little patient-centred communication. Others explained that they were too ill to take in information offered to them.

'I think people need to be told what's happening to them, like now and the near future will be like this or like that, but to be told is the best thing in life.' [ 68 yr transplant survivor, HFNO $\times 5$ days]

'There was too much commotion down there [in the high care wards]. Nobody seemed to communicate right with each other. Take this, put this there. They were like, push that one there, you know... I didn't understand, because I was very sick, but I was happy to see them around me every day.' [ $35 \mathrm{yr}$ woman, $\mathrm{HFNO} \times 3$ days] 


\section{Risks for severe COVID}

Many participants were unclear on the risks for severe COVID, and felt mystified that they required hospitalisation when relatives and friends were asymptomatic or mildly ill. Many expressed shock that they had contracted COVID and had become seriously ill.

'That's why I ask myself, why me? A lot of people, why it chooses me.' [54 yr woman diabetic and hypertensive]

'Never in my entire life, I thought this would happen to me.' [59 yr man, hypertensive with previous myocardial infarction]

'No, I didn't believe. I see other people. I say it's not for me, it's for them.' [48 yr man, diabetic and HIV positive]

One participant felt that COVID and the risks for severe disease were poorly explained to the public.

'The government didn't explain. No. The people, they don't understand what is going on. Even diabetics. No, to be honest, to me I start to take it seriously here.' [67 yr diabetic and hypertensive]

\section{Optimism for the future}

All participants felt that they had new focus or inspiration in their lives and felt optimistic about the future. Many were proud to have survived this illness and were excited to go home, reunite with their families, and make changes to their lifestyle.

'This was a tough experience. So, I must look forward now.' [57 yr seamstress, HFNO $\times 9$ days]

'I will never take life for granted. Never again, but I've learned a lot of lessons. It's like everything is new to me, because I've experienced a lot of pain, but now I feel better. Everything of mine is better. I'm coping.' [34 yr cleaner, ventilated $\times 5$ days]

All participants expressed the belief that their lives would change. All felt that their severe illness was a call to take better care of themselves.

I'm not getting any younger, 67 is a big age and I'm thankful that God spared me, but this is also an eye opener that God gave me. It's time you look after yourself.' [67 yr pensioner, $\mathrm{HFNO} \times 4$ days]

Some intended to change their diet and lose weight, while many pledged better adherence to healthcare appointments and medication, and to spending more time with family.

'Take more care of my health. I'm going to focus more to close relatives, family, everyone that is dear to me because this is the situation.' [45 $\mathrm{yr}$ electrician, $\mathrm{HFNO} \times 5$ days]

One participant explained that he felt compelled to follow earlier ambitions that had been set aside.

'I took time to think a lot about my life and now I just go back to things that I left behind and never finished. I still got a lucky .... and God gave me a lot of talent. Which I wasn't using for many years, because I got distracted, but now I've got the time.' [61 yr musician, $\mathrm{HFNO} \times 6$ days $]$

\section{Discussion}

The impetus for this study was a desire to develop a better understanding of the lived experience of severe COVID-19 illness in an urban South African community - thus providing greater insight into unmet needs and areas for improvement. The major factors reported by the participants were the distressing nature of their experience characterised by shortness of breath, loneliness and helplessness, and faith-based beliefs seeing them through, with tremendous gratitude to HCWs. Most participants reported a better understanding of COVID and how dangerous it was and expressed optimism towards the future, with resolve to implement lifestyle changes.

Our participants' descriptions of critical COVID-19 demonstrate the anxiety, debilitating dyspnoea, loneliness and confusion experienced during a critical illness with an uncertain outcome. Many shared powerful imageries of their ordeals. Many participants described their lack of control as a major issue, similar to insights shared by critical COVID-19 survivors elsewhere. ${ }^{[6,11,12]}$ Countering this, faith-based beliefs were an important source of comfort to many participants, together with confidence in the medical teams.

Silent hypoxia, or lack of discomfort at very low blood-oxygen concentrations, is well described in COVID-19. ${ }^{[13]}$ However, for many patients in our study, dyspnoea was a major cause of discomfort and distress. In COVID-19, dyspnoea may be multifactorial. Anxiety contributes to an unpleasant feeling of air hunger, and tends to occur early in the disease as the first symptoms emerge. ${ }^{[15]}$ Acute respiratory distress syndrome (ARDS) or pulmonary thrombosis causes hypoxaemia, which may cause dyspnoea and typically occurs several days after the onset of the first COVID symptoms. ${ }^{[14]}$ Our study, and others, suggests that psychotherapy might be a useful adjunct to medical treatment. ${ }^{[16]}$

All participants expressed gratitude and satisfaction at the level of care received at the hospital. This is a testimony to the commitment of all staff working under very difficult and stressful circumstances, and underscores our HCWs' skill, sincere care and kindness. Our participants' testimonies remind us that good, clear communication with critically ill participants needs to remain a priority, despite the challenges including dealing with anxiety-stricken participants with a low capacity for absorbing information, and overwhelming participant numbers. As writer Maya Angelou said: 'I've learned that people will forget what you said, people will forget what you did, but people will never forget how you made them feel. ${ }^{\text {[17] }}$

Adults of any age can develop severe COVID-19 disease, but older age ( $\geq 64$ years) is a major risk factor for progression to ARDS. Other comorbidities including obesity, poorly controlled diabetes mellitus, hypertension and pre-existing chronic cardiac, renal and pulmonary conditions, together with malignancies and untreated human immunodeficiency virus (HIV) infection, infer an increased risk of severe disease and death. ${ }^{[18]}$ Of great concern is that the vast majority of participants in the present study were unaware of these risk factors and, although 18 of the 21 participants themselves had one or more risk factors, most expressed surprise that they had been very ill. This is an area for better public health education, because improved understanding of the virus and one's vulnerabilities may improve adherence with recommendations to avoid infection. Elsewhere, compliance with restrictions imposed to reduce the spread of COVID-19 infections has been shown to be complex, with a need for presentation of evidence on the effectiveness and reasons behind measures. ${ }^{[19]}$ 
Gratifyingly, upon discharge from hospital, the vast majority of participants understood their vulnerability to severe COVID disease, and many felt motivated to discuss their experiences with the family and community. In addition, many participants felt inspired to address unhealthy lifestyle factors and improve control of their chronic illnesses, suggesting psychological growth. The motivation to focus on selfcare and invest in fulfilling relationships is a positive outcome from a difficult and terrifying ordeal. Similar positive cues to action regarding improved health knowledge, lifestyle and care-seeking behaviour owing to the COVID pandemic are reported by others. ${ }^{[5,6,20]}$

Some limitations of the study include the cross-sectional nature of the study done in a tertiary care setting, and the exclusion of participants who were not able to converse in English or Afrikaans. Further, interviews took place in the general COVID wards, with the interviewer wearing full personal protective equipment, and this may have hindered communication. A private, quiet space would have been a preferable setting but was logistically impossible owing to infection control measures and ongoing oxygen requirements of recovering participants. In addition, many participants were short of breath and fatigued from their illness, which may have blunted their responses. At the time of the study in mid-2020, COVID-19 vaccines were not yet available, and therefore discussions about vaccination were not included in the interviews.

\section{Conclusion}

The use of qualitative methods to understand participants' reactions to severe COVID-19 illness adds a dimension to our understanding of how experiencing a severe illness affects the person not just physically, but also psychologically. These experiences were reported by the participants as life changing. These findings contribute to understanding ways of improving services to ensure that the trauma experienced by such participants is minimised. Future interventions might include offering a follow-up debriefing session on discharge from high care wards or ICU, and support for lifestyle changes that patients expressed the desire to implement.

\section{Declaration. None.}

Acknowledgements. The authors are grateful to the patients who participated in this study, frequently under difficult circumstances, many of whom were prepared to converse in English or Afrikaans which were not their first languages. We also thank Mrs Amelia Lawrence for transcription and translation.

Author contributions. BH and MS analysed and interpreted the transcribed audio-recordings. All authors contributed to, read and approved the final manuscript.

Funding. None.

Conflicts of interest. None.
1. Calligaro GL, Lalla U, Audley G, et al. The utility of high-flow nasal oxygen for severe COVID-19 pneumonia in a resource-constrained setting: A multi- centre prospective observational study. EClinicalMedicine 2020;28:100570. https://doi.org/10.1016/j. eclinm.2020.100570

2. Richardson S, Hirsch JS, Narasimhan M, et al. Presenting characteristics, comorbidities, and outcomes among 5700 patients hospitalised with COVID-19 in the New York city area. JAMA 2020;323(20):2052-2059. https://doi.org/10.1001/jama.2020.6775

3. Yang X, Yu Y, Xu J, et al. Clinical course and outcomes of critically ill patients with SARS CoV-2 pneumonia in Wuhan, China: A single-centered, retrospective, observational study. Lancet Respir Med 2020;8(5):475-481. https://doi.org/10.1016/s2213-2600(20)30079-5

4. Mendelson M, Boloko L, Boutall A, et al. Clinical management of COVID-19: Experiences of the COVID-19 epidemic from Groote Schuur Hospital, Cape Town, South Africa. S Afr Med J 2020;110(10):973-981. https://doi.org/10.7196/samj.2020.v110i10.15157

5. Sun N, Wei L, Wang H, et al. Qualitative study of the psychological experience of COVID-19 patients during hospitalisation. J Affect Disord 2021;278:15-22. https://doi. org/10.1016/j.jad.2020.08.040

6. Missel M, Bernild C, Westh Christensen S, Dagyaran I, Kikkenborg Berg S. The marked body - a qualitative study on survivors embodied experiences of a COVID-19 illness trajectory. Scand J Caring Sci 2021 (epub 18 March 2021). https://doi.org/10.1111/ scs. 12975

7. Norouzadeh R, Abbasinia M, Tayebi Z, et al. Experiences of patients with COVID-19 admitted to the intensive care units: A qualitative study. J Patient $\operatorname{Exp} 2021 ; 8$. https://doi. org/10.1177/23743735211007359

8. Rees S, Griffiths F, Bassford C, et al. The experiences of health care professionals, patients, and families of the process of referral and admission to intensive care: A systematic literature review. J Intensive Care Soc 2020;21(1):79-86. https://doi.org/10.1177/1751143719832185

9. Scheunemann LP, White JS, Prinjha S, et al. Post- intensive care unit care. A qualitative analysis of patient priorities and implications for redesign. Ann Am Thorac Soc 2020;17(2):221-228. https://doi.org/10.1513/annalsats.201904-332oc

10. Teti M, Schatz E, Liebenberg L. Methods in the time of COVID-19: The vital role of qualitative inquiries. Int J Qualitat Methods 2020;19:1-5. https://doi. org/10.1177\%2F1609406920920962

11. Ramachandran S. A junior doctor's experience of critical illness: From treating patients to becoming a patient with COVID-19. Anaesth Rep 2020;8:59-62. https://doi.org/10.1002/ anr3.12052

12. Sahoo S, Mehra A, Suri V, et al. Lived experiences of COVID-19 intensive care unit survivors. Indian J Psychol Med 2020;42(4):387-390. https://doi.org/10.1177\%2F0253717620933414

13. Tobin MJ, Laghi F, Jubran A. Why COVID-19 silent hypoxemia is baffling to physicians. Am J Respir Crit Care Med 2020;202(3):356-360. https://doi.org/10.1164/rccm.202006 $2157 \mathrm{cp}$

14. Nouri-Vaskeh M, Sharifi A, Khalili N, Zand R, Sharifi A. Dyspneic and non-dyspneic (silent) hypoxemia in COVID-19: Possible neurological mechanism. Clin Neurol Neurosurg 2020;198:106217. https://doi.org/10.1016/j.clineuro.2020.106217

15. Cohen PA, Hall LE, John JN, Rapoport AB. The early natural history of SARS-CoV-2 infection: Clinical observations from an urban, ambulatory COVID-19 clinic. Mayo Clin Proc 2020;95(6):1124-1126. https://doi.org/10.1016\%2Fj.mayocp.2020.04.010

16. Khawam E, Khouli H, Pozuelo L. Treating acute anxiety in patients with COVID-19. Cleve Clin J Med 2020;18(12) (epub 1 December 2020). https://doi.org/10.3949/ccjm.87a. ccc016

17. The Arts Go To School: Classroom-based activities that focus on music, painting, drama, movement, media, and more. Hachiya DBaM, ed. Pembroke Publishers, Markham, 2004.

18. Boulle A, Davies MA, Hussey H, et al. Risk factors for coronavirus disease 2019 (COVID-19) death in a population cohort study from the Western Cape Province, South Africa. Clin Infect Dis 2021;73(7):e2005-e2015. https://doi.org/10.1093/cid/ciaal198

19. Zimmermann BM, Fiske A, McLennan S, Sierawska A, Hangel N, Buyx A. Motivations and limits for COVID-19 policy compliance in Germany and Switzerland. Int J Health Policy Manag 2021 (epub 21 April 2021). https://doi.org/10.34172/ijhpm.2021.30

20. Saah FI, Amu H, Seidu AA, Bain LE. Health knowledge and care seeking behaviour in resource-limited settings amidst the COVID-19 pandemic: A qualitative study in Ghana. PLoS ONE 2021;16(5):e0250940. https://doi.org/10.1371\%2Fjournal.pone.0250940 